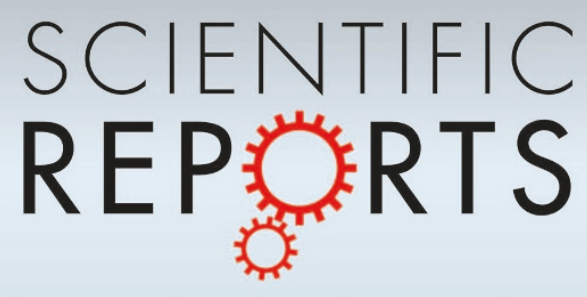

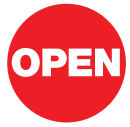

SUBJECT AREAS: HETEROGENEOUS

CATALYSIS

ASTEROIDS, COMETS AND KUIPER BELT

INTERSTELLAR MEDIUM

METEORITICS

Received

18 October 2012

Accepted

5 February 2013

Published

26 February 2013

Correspondence and requests for materials should be addressed to

S.C. (cazaux@astro. rug.nl)

\section{How micron-sized dust particles determine the chemistry of our Universe}

\author{
François Dulieu', Emanuele Congiu', Jennifer Noble², Saoud Baouche' ', Henda Chaabouni', \\ Audrey Moudens' ', Marco Minissale' \& Stéphanie Cazaux ${ }^{3}$
}

'LERMA Université de Cergy-Pontoise, Observatoire de Paris, ENS, UPMC 8112 du CNRS, 5 mail Gay Lussac, 95000 Cergy Pontoise Cedex, France, ${ }^{2}$ Aix-Marseille Université, PIIM UMR 7345, 13397, Marseille, France, ${ }^{3}$ Kapteyn Astronomical Institute, PO box 800,9700 AV Groningen, The Netherlands.

In the environments where stars and planets form, about one percent of the mass is in the form of micro-meter sized particles known as dust. However small and insignificant these dust grains may seem, they are responsible for the production of the simplest $\left(\mathrm{H}_{2}\right)$ to the most complex (amino-acids) molecules observed in our Universe. Dust particles are recognized as powerful nano-factories that produce chemical species. However, the mechanism that converts species on dust to gas species remains elusive. Here we report experimental evidence that species forming on interstellar dust analogs can be directly released into the gas. This process, entitled chemical desorption (fig. 1), can dominate over the chemistry due to the gas phase by more than ten orders of magnitude. It also determines which species remain on the surface and are available to participate in the subsequent complex chemistry that forms the molecules necessary for the emergence of life.

ighty years ago, dust was first identified in the interstellar space as the "something" which is obscuring
the light coming from stars'. For several decades, dust grains were thought to be only passive and
annoying entities present towards any astrophysical objects that astronomers wanted to observe. Only in the $60 \mathrm{~s}$, the real nature of dust was revealed and it appeared that dust is a powerful interstellar catalyst ${ }^{2}$. The presence of several chemical species that cannot form efficiently in the gas only highlighted the need to explore the chemistry occurring on interstellar dust. At first, the use of chemistry occurring on dust to palliate the failure of gas phase chemistry was qualified as the refuge of the scoundrels ${ }^{3}$. However, while the role of dust as interstellar catalyst is now well recognized, the mechanism that allows the formed species on the surface to populate the gas phase is still unknown. This is a real paradox and issue of modern astrochemistry: to reconcile almost non-observable solid phase dust catalysis to observable products in the gas phase. Observations of deeply embedded protostars show that the disappearance of $\mathrm{CO}$ in the gas (because $\mathrm{CO}$ sticks on the dust) leads to a high abundance of $\mathrm{CH}_{3} \mathrm{OH}$ in the gas ${ }^{4}$. This anti-corelation demonstrates that $\mathrm{CO}$ converts into $\mathrm{CH}_{3} \mathrm{OH}$ on dust and is released into the gas phase through an efficient mechanism. It appears that many reactions occurring on dust grains are accompanied with an excess energy which is too large to dissipate. Consequently, the formed species are ejected in the gas phase upon formation. This process, called chemical desorption, implies that the chemistry occurring on interstellar dust affects the composition of the gas. Whereas the existence of this process, first suggested one decade $a^{g} o^{5}$, has been shown theoretically $y^{6-10}$, it has been experimentally observed only for $\mathrm{H}_{2}$ molecules ${ }^{11-14}$ which is the most favorable expected case (very light, weakly bounded, very exothermic). In this work, we present the first experimental evidence of this process for several reactions occurring on dust analogs. We show that chemical desorption is very important (more than $90 \%$ of water formed through $\mathrm{OH}+\mathrm{H}$ on the dust is released in the gas phase) and has been considerably underestimated by previous assumptions. We discuss the importance of this mechanism in comparison with the chemistry in the gas, and show that it can dramatically change the chemical composition of the interstellar medium. In this sense, because the chemical composition of a medium is one of the main observational tool used to determine physical conditions, we show that current astrochemical models have to account for the catalytic role of dust due to chemical desorption in order to properly describe/predict observations. This new result also affects our current understanding of star formation. By changing the abundances of molecules present in the gas of a cloud that undergoes gravitational collapse to form a star, the gas cools more or less rapidly, which impacts the efficiency and characteristics (mass, binarity) of star formation. 


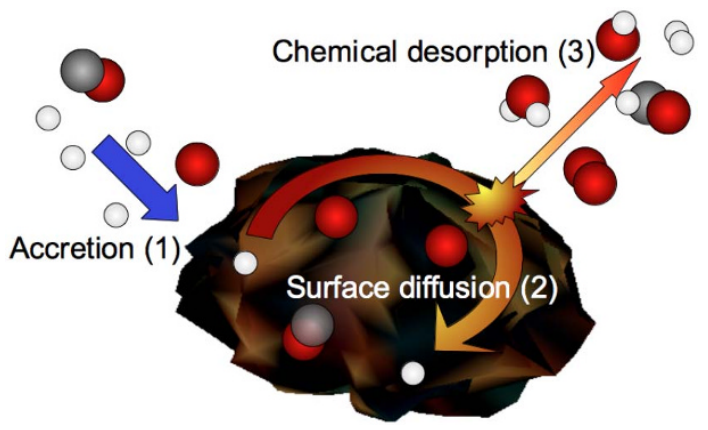

Figure $1 \mid$ Sketch that illustrates the chemical desorption process. Species coming from the gas accrete on the dust surface and can meet each other to form other species. For some reactions shown in this study, the formed product is ejected in the gas.

\section{Results}

We study experimentally the formation of water on silicate surfaces. For this purpose, we deposit $\mathrm{O}_{2}$ molecules on silicates, and deposit $\mathrm{D}$ atoms in a second step. The surface chosen in this study is usually used as interstellar dust analogs. After deposition at $10 \mathrm{~K}$, the surface temperature is increased up to $200 \mathrm{~K}$ and molecules are measured in the gas with a mass spectrometer. The results of these experiments are presented in fig. 2 . The solid lines represent the thermal desorption spectra of $\mathrm{O}_{2}$ (mass 32), the dashed lines the signal for $\mathrm{D}_{2} \mathrm{O}$ (mass 20), while dotted lines the signal of $\mathrm{D}_{2} \mathrm{O}_{2}$ (mass 36), as the temperature of the surface is increased at $0.04 \mathrm{~K} / \mathrm{s}$. The thermal desorption spectra of the 3 different species on silicate surfaces are represented in red. For comparison, we also report our experimental results for the formation of water on graphite (green), and non porous ice (blue).

The black line represents the thermal desorption spectrum when only $\mathrm{O}_{2}$ is deposited on silicate surfaces. It desorbs as a single peak at around $35 \mathrm{~K}$ and the integrated signal corresponds to 1.0 monolayer $\left(1 \mathrm{ML} \sim 10^{15}\right.$ atoms $/ \mathrm{cm}^{2}$ ). The red lines (solid for $\mathrm{O}_{2}$, dashed for $\mathrm{D}_{2} \mathrm{O}$ and dotted for $\mathrm{D}_{2} \mathrm{O}_{2}$ ) represent the thermal desorption spectra when $\mathrm{O}_{2}$ is similarly deposited, and is exposed to $410^{15}$ atoms $/ \mathrm{cm}^{2}$ of
Table 1 | TPD desorption peaks and associated binding energies of the molecules on the silicate surface

\begin{tabular}{lccc} 
Species & TPD peak & Binding energy $(\mathrm{K})$ & ref. \\
\hline $\mathrm{H}_{2}$ & $12 \mathrm{~K}$ & 300 & {$[11]$} \\
$\mathrm{H}$ & & 500 & {$[20]$} \\
$\mathrm{O}$ & 1100 & This work \\
$\mathrm{O}_{2}$ & $40 \mathrm{~K}$ & 1255 & {$[15]$} \\
$\mathrm{O}_{3}$ & $67 \mathrm{~K}$ & 2100 & $\begin{array}{c}\text { Minisale et al. } \\
\text { submitted }\end{array}$ \\
$\mathrm{O}_{2} \mathrm{H}$ & & 4000 & \\
$\mathrm{OH}$ & $140 \mathrm{~K}$ & 4600 & This work and [21,22] \\
$\mathrm{H}_{2} \mathrm{O}$ & $145 \mathrm{~K}$ & 4800 & This work \\
$\mathrm{H}_{2} \mathrm{O}_{2}$ & $180 \mathrm{~K}$ & 6000 & \\
\hline
\end{tabular}

D. In this case, the desorption of $\mathrm{O}_{2}$ around $35 \mathrm{~K}$ is very weak compared to the deposition of $\mathrm{O}_{2}$ only, meaning than most $(\geq 90 \%)$ of the initial $\mathrm{O}_{2}$ molecules have disappeared. $\mathrm{D}_{2} \mathrm{O}$ and $\mathrm{D}_{2} \mathrm{O}_{2}$ desorption features are observed at $150 \mathrm{~K}$, and before $200 \mathrm{~K}$, respectively. The location of these peaks in temperature allow to derive the binding energies of the different species on the substrate. These binding energies are reported in table 1. Previous studies showed that $\mathrm{O}_{2}$ reacts quickly with $\mathrm{D}$, forming $\mathrm{O}_{2} \mathrm{D}, \mathrm{D}_{2} \mathrm{O}_{2}$ and finally $\mathrm{D}_{2} \mathrm{O}^{16-18}$. The area of the desorbed peaks indicates that only $35 \%$ of the oxygen has been included in the $\mathrm{D}_{2} \mathrm{O}$ and $\mathrm{D}_{2} \mathrm{O}_{2}$ molecules, meaning than around $65 \%$ of the $\mathrm{O}_{2}$ molecules are missing from the surface.

The $\mathrm{O}_{2}$ molecules missing in the desorption spectra are believed to be ejected into the gas phase during exposure with $\mathrm{D}$ atoms, following chemical reaction. In this sense, once new species are formed, and are unable to thermalize on the surface, they are released into the gas phase (the so called chemical desorption). To prove the existence of this process, we monitor the mass signal during $\mathrm{D}$ irradiation, with the quadrupole mass spectrometer placed in a position remote from the sample. The measurements are reported in the inset of fig. 2 before and during $\mathrm{D}$ irradiation for $\mathrm{D}_{2} \mathrm{O}$ (mass 20), $\mathrm{DO}_{2}$ (mass 34) and $\mathrm{D}_{2} \mathrm{O}_{2}$ (mass 36). We observe a direct $\mathrm{D}_{2} \mathrm{O}$ signal far above the

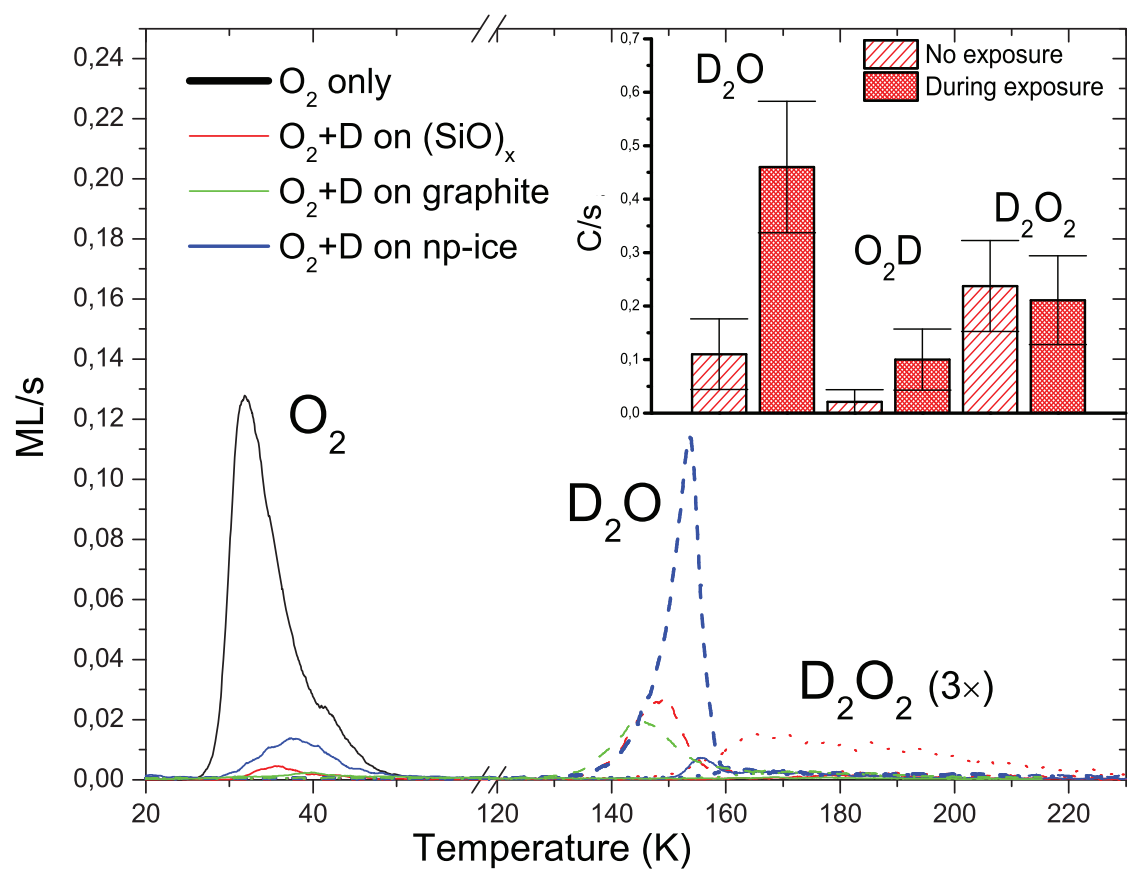

Figure 2 Experimental evidence of chemical desorption. Desorption peaks of $\mathrm{O}_{2}$ (solid), $\mathrm{D}_{2} \mathrm{O}$ (dashed) and $\mathrm{D}_{2} \mathrm{O}_{2}$ (dotted) species after the exposure of $\mathrm{D}$ atoms on $1 \mathrm{ML}$ coverage of $\mathrm{O}_{2}$ ice deposited on different substrates (silicates $\mathrm{SiOx}$ in red, graphite in green and np-ASW ice in blue) held at $10 \mathrm{~K}$. Inset: $\mathrm{D}_{2} \mathrm{O}, \mathrm{D}_{2} \mathrm{O}_{2}$ and $\mathrm{D}_{2} \mathrm{O}$ monitored with the QMS during the exposure of $\mathrm{D}$ atoms on $1 \mathrm{ML}$ coverage of $\mathrm{O}_{2}$ ice deposited on the silicate substrate at $10 \mathrm{~K}$. 
noise level, indicating that $\mathrm{D}_{2} \mathrm{O}$ is chemically desorbed. On the other hand, we do detect a very small increase of the $\mathrm{DO}_{2}$ signal (in the noise) but no increase of the $\mathrm{D}_{2} \mathrm{O}_{2}$ signal. $\mathrm{OD}$ (mass 18) and $\mathrm{O}_{2}$ (mass 32) are also monitored, but the presence of $\mathrm{H}_{2} \mathrm{O}$ (mass 18) and $\mathrm{O}_{2}$ as minor contaminants in the vacuum decreases the signal to noise ratio, allowing only a one sigma detection of $\mathrm{O}_{2}$. The measurement before/during irradiation provides clear evidence of the chemical desorption, and that the product concerns mainly $\mathrm{D}_{2} \mathrm{O}$. The signal before and during irradiation cannot be directly compared since the QMS is not located at the same place during these experiments. For the TPDs, it is placed in front of the sample to maximize the signal. In the irradiation configuration, it is placed closed to the surface, but with an angle of about $45^{\circ}$.

Our experimental results show that in the case of water formation on a silicate substrate, $65 \%$ of the $\mathrm{O}_{2}$ molecules initially on the substrate are no longer present after $\mathrm{D}$ irradiation. By measuring the amount of species desorbing before/during the irradiation of $\mathrm{O}_{2}$ with $\mathrm{D}$ atoms, we show that repetitive addition of $\mathrm{D}$ to $\mathrm{O}_{2}$ leads to the detection of $\mathrm{D}_{2} \mathrm{O}$ in the gas phase. The possible reactions routes are the following:

reaction $1: \mathrm{O}_{2}+\mathrm{D} \rightarrow \mathrm{DO}_{2}+\mathrm{D} \rightarrow \mathrm{D}_{2} \mathrm{O}_{2}+\mathrm{D} \rightarrow \mathrm{D}_{2} \mathrm{O}+\mathrm{OD} \quad k$
reaction2: $\mathrm{O}_{2}+\mathrm{D} \rightarrow \mathrm{DO}_{2}+\mathrm{D} \rightarrow \mathrm{OD}+\mathrm{OD}$

The branching ratio of these two reactions are $k$ and $(1-k)$. The table 2 reports the reactions involved in our experiment as well as their exothermicities. The reaction $\mathrm{O}_{2}+\mathrm{D}$ leading to $\mathrm{DO}_{2}$ is slightly exothermic $(2.3 \mathrm{eV})$ but we only see a slight increase of the mass signal (mass 34) when $\mathrm{O}_{2}$ is exposed to $\mathrm{D}$ atoms (this signal is in the noise). The reaction $\mathrm{DO}_{2}+\mathrm{D}$ leading to $\mathrm{D}_{2} \mathrm{O}_{2}$ has an exothermicity of $3.7 \mathrm{eV}$, but $\mathrm{D}_{2} \mathrm{O}_{2}$ is not detected in the gas phase during irradiation. Previous studies showed that $\mathrm{H}_{2} \mathrm{O}_{2}\left(\mathrm{D}_{2} \mathrm{O}_{2}\right)$ can easily decompose in $\mathrm{H}_{2} \mathrm{O}\left(\mathrm{D}_{2} \mathrm{O}\right)$ on the wall of chamber ${ }^{19}$, meaning that the $\mathrm{H}_{2} \mathrm{O}\left(\mathrm{D}_{2} \mathrm{O}\right)$ signal obtained is due to molecules bouncing on the wall. In our experimental configuration, the number of bounced molecules that are detected are less than $10 \%$ (see Ref. 13), and can be neglected in our analysis. We know that $\mathrm{D}_{2} \mathrm{O}_{2}$ leaving in the gas phase cracks into $\mathrm{D}_{2} \mathrm{O}$ (for $\sim 40 \%$ ) but that $\sim 60 \%$ stays under the form of $\mathrm{D}_{2} \mathrm{O}_{2}$. The cracking pattern of $\mathrm{D}_{2} \mathrm{O}_{2}$ is estimated during the TPD after $160 \mathrm{~K}$, where we see a signal at the masses $20\left(\mathrm{D}_{2} \mathrm{O}\right)$ and 36 $\left(\mathrm{D}_{2} \mathrm{O}_{2}\right)$ simultaneously. Therefore, if $\mathrm{D}_{2} \mathrm{O}_{2}$ is chemically desorbed into the gas phase, we should see an increased of the signal during irradiation. The reaction $\mathrm{DO}_{2}+\mathrm{D}$ can also lead to $\mathrm{OD}+\mathrm{OD}$, and has an exothermicity of $1.6 \mathrm{eV}$. However, we are not able to detect $\mathrm{OD}$ molecules since their mass coincide with $\mathrm{H}_{2} \mathrm{O}$ (mass 18) which is contaminating the mass spectra. We are therefore not able to derive the fraction that is chemically desorbed for this reaction. The reaction $\mathrm{D}_{2} \mathrm{O}_{2}+\mathrm{D} \rightarrow \mathrm{D}_{2} \mathrm{O}+\mathrm{OD}$ is exothermic with an energy of $3.1 \mathrm{eV}$, and the reaction $\mathrm{OD}+\mathrm{D} \rightarrow \mathrm{D}_{2} \mathrm{O}$ has an exothermicity of $5.2 \mathrm{eV}$. Since $\mathrm{D}_{2} \mathrm{O}$ is observed in the gas phase during exposition of $\mathrm{O}_{2}$ with $\mathrm{D}$ atoms, we can not differentiate which of these two routes is responsible for the important chemical desorption of $\mathrm{D}_{2} \mathrm{O}$ which is observed.

\begin{tabular}{|lcc|} 
Table $2 \mid$ Chemical desorption efficiency of different reactions \\
Reaction & Exothermicity (eV) & $\begin{array}{c}\text { Fraction released } \\
\text { in gas phase }\end{array}$ \\
\hline $\mathrm{OD}+\mathrm{D} \rightarrow \mathrm{D}_{2} \mathrm{O}$ & 5.2 & $>90 \%$ \\
$\mathrm{O}_{2}+\mathrm{D} \rightarrow \mathrm{DO} \mathrm{O}_{2}$ & 2.3 & $<10 \%$ \\
$\mathrm{DO}_{2}+\mathrm{D} \rightarrow \mathrm{D}_{2} \mathrm{O}_{2}$ & 3.7 & $<10 \%$ \\
$\mathrm{DO}_{2}+\mathrm{D} \rightarrow \mathrm{OD}+\mathrm{OD}$ & 1.6 & $<10 \%$ \\
$\mathrm{D}_{2} \mathrm{O}_{2}+\mathrm{D} \rightarrow \mathrm{D}_{2} \mathrm{O}+\mathrm{OD}$ & 3.1 & $60 \%$ Duliev et al. in prep. \\
$\mathrm{O}+\mathrm{O} \rightarrow \mathrm{O}_{2}$ & 5.2 & $\leq 10 \%$ Minissale et al. in \\
$\mathrm{O}+\mathrm{O}_{2} \rightarrow \mathrm{O}_{3}$ & 1.1 & prep. \\
& &
\end{tabular}

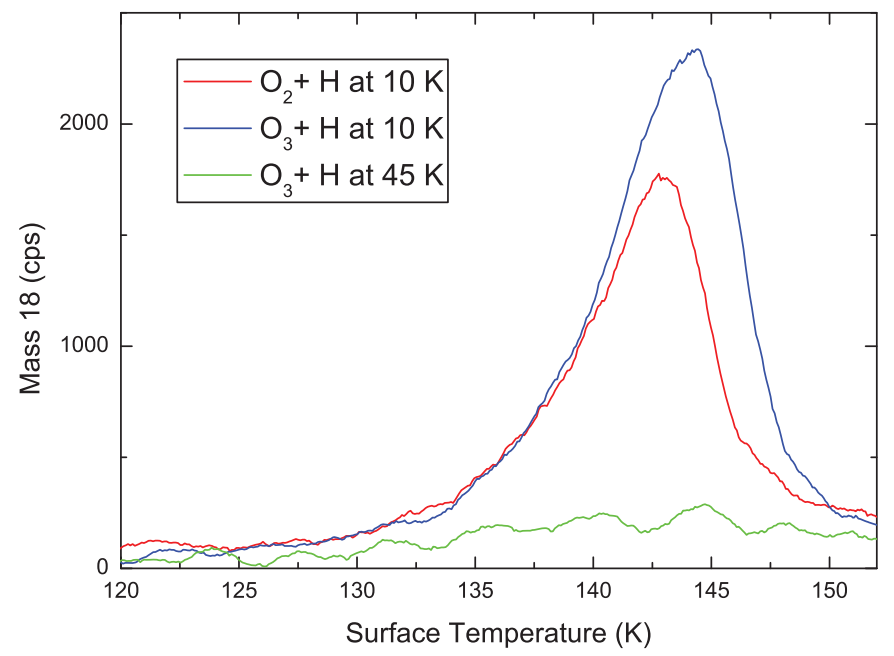

Figure 3 Desorption spectra of water after its formation via $1 \mathrm{ML}$ of $\mathrm{O}_{2}$ followed by $710^{15} \mathrm{H} / \mathrm{cm}^{2}$ on silicates held at $10 \mathrm{~K}$ (blue); $1 \mathrm{ML}$ of $\mathrm{O}_{3}$ followed by $710^{15} \mathrm{H} / \mathrm{cm}^{2}$ atoms on a surface held at $10 \mathrm{~K}$ (red) and at $45 \mathrm{~K}$ (green).

We perform complementary TPD experiments to address the origin of the very important chemical desorption of $\mathrm{D}_{2} \mathrm{O}$. Here we use hydrogen instead of deuterium. Figure 3 represents the desorption spectra of water after its formation via $10^{15}$ atoms $/ \mathrm{cm}^{2}$ of $\mathrm{O}_{2}$ followed by $7 \times 10^{15}$ atoms $/ \mathrm{cm}^{2}$ of $\mathrm{H}$ atoms on a surface held at $10 \mathrm{~K}$ (blue); $10^{15}$ atoms $/ \mathrm{cm}^{2}$ of $\mathrm{O}_{3}$ followed by $7 \times 10^{15}$ atoms $/ \mathrm{cm}^{2}$ of $\mathrm{H}$ atoms on a surface held at $10 \mathrm{~K}$ (red) and $45 \mathrm{~K}$ (green). We can see that in the case of $\mathrm{O}_{3}$, there is a lower production of $\mathrm{H}_{2} \mathrm{O}$, because the formation of water is made of two sub-routes $\mathrm{O}_{3}+\mathrm{H} \rightarrow \mathrm{OH}+\mathrm{O}_{2}$. Performing experiments at $45 \mathrm{~K}$, where $\mathrm{O}_{2}$ is evaporating at a high rate, almost forbid the $\mathrm{O}_{2}+\mathrm{H}$ sub-route, so the $\mathrm{O}_{3}+\mathrm{H}$ at $45 \mathrm{~K}$ is almost like observing the $\mathrm{OH}+\mathrm{H}$ reaction. We can see clearly that there is almost no water produced that way, indicating a very strong chemical desorption during exposure through the reaction $\mathrm{OH}+\mathrm{H}$. To check that the disappearance of the water signal for $\mathrm{O}_{3}+\mathrm{H}$ at $45 \mathrm{~K}$ is not due to the fact that the reaction does not proceeds, we measure the amount of $\mathrm{O}_{3}$ present on the surface as $\mathrm{O}_{3}$ is exposed to $\mathrm{H}$ atoms. We see that the peak of $\mathrm{O}_{3}$, located at $67 \mathrm{~K}$ in the TPD, decreases as the exposure with $\mathrm{H}$ atoms increases. This shows that even if the residence time of $\mathrm{H}$ atoms on the surface is short at $45 \mathrm{~K}$, it is long enough to proceed radical-radical reactions, as observed here for the reaction $\mathrm{O}_{3}+\mathrm{H}$ and also observed for $\mathrm{NO}+\mathrm{H}^{23}$. To summarize, we show that the $\mathrm{OH}+\mathrm{H}$ route is the one responsible for the important amount of water during exposure of $\mathrm{O}_{2}$ with $\mathrm{H}$ or D. Since most of the water formed through this route is ejected in the gas phase, this allows us to bring further constrains on our first TPD experiment. The reaction $\mathrm{O}_{2}+\mathrm{D}$ leads to the formation of $\mathrm{D}_{2} \mathrm{O}_{2}$ and then $\mathrm{D}_{2} \mathrm{O}+$ $\mathrm{OD}$, or to the formation of $2 \mathrm{OD}$. Since OD on the surface reacts with a $\mathrm{D}$ to form $\mathrm{D}_{2} \mathrm{O}$ and is ejected in the gas, $\mathrm{D}_{2} \mathrm{O}$ and $\mathrm{D}_{2} \mathrm{O}_{2}$ observed in the TPD are the results of the reaction 1 . This implies that the $35 \%$ of $\mathrm{O}_{2}$ found on the surface come from the reaction 1 , and that the branching ratio is $\mathrm{k}=0.35$. The reactions occurring in our experiment, as well we the fraction of species chemically desorbed are reported in table 2 .

Similar experiments have been performed on graphitic surface and on water ice substrate (Dulieu et al. in prep). For graphite, $80 \%$ of the $\mathrm{O}_{2}$ molecules initially on the substrate are no longer present after D exposure. For the water ice as a substrate, this percentage strongly depends on the morphology of the ice, and can vary from almost $0 \%$ in the case of porous ice, to $40 \%$ in the case of non porous amorphous ice. These percentages are the fraction of the newly formed species that are ejected into the gas phase upon formation due to the high 
exothermicity of the reaction. Coupling to the substrate (phonons) is believed to be the source of the different rates observed for different substrates. Therefore, our results show (table 2) that the fraction of species released into the gas phase upon formation do not depend directly on the exothermicity of the reaction and on the binding energy of the products. The mass of the formed species also plays a fundamental role, as well as the type of substrate that is considered. Many follow-up experimental studies using different reactions/substrates are envisaged to understand the nature of the chemical desorption process.

\section{Discussion}

To estimate the impact of chemical desorption on the gas phase composition of astrophysical environments, we use a theoretical model ${ }^{10,24}$, but include the chemical desorption yields derived from the experiments presented in the previous section. In this sense, we quantify the direct impact of the chemistry on dust on the gas phase. For some reactions occurring on the surface, an important amount of the formed species are ejected in the gas phase. Therefore, within the parameter range at which these reactions dominate the chemistry on the dust surface, an important contribution in the gas phase is expected. Figure 4 illustrates the chemical desorption of $\mathrm{H}_{2} \mathrm{O}, \mathrm{OH}$ and $\mathrm{O}_{2}$ as a function of the dust temperature. The calculations of these efficiencies are reported in the methods section. These efficiencies are the amount of $\mathrm{O}$ that is converted to $\mathrm{OH}, \mathrm{H}_{2} \mathrm{O}$ and $\mathrm{O}_{2}$ on the dust and directly released into the gas phase. In this sense, the calculated efficiencies directly provide the conversion rate of gas phase atomic oxygen into another gas phase species by the use of grain surface chemistry. As $\mathrm{OH}$ is formed through $\mathrm{O}+\mathrm{H}, \mathrm{O}_{2}$ through $\mathrm{O}+$ $\mathrm{O}$, or water through $\mathrm{OH}+\mathrm{H}$, an important fraction of the newly formed molecules is released into the gas phase. The formation efficiency is on the order of $\sim 30 \%$ for $\mathrm{OH}$ (below $15 \mathrm{~K}$ ) and $\sim 70 \%$ for water (below $\sim 25 \mathrm{~K}$ ). For $\mathrm{O}_{2}$, there are two main temperature ranges where desorption into the gas phase is important. The first one, located around $17 \mathrm{~K}$, has an efficiency of about $4 \%$, while the second one, located around $\sim 35 \mathrm{~K}$, has an efficiency of $0.2 \%$. If a strong UV field is considered in our calculations, then the species on the surface can be photo-dissociated and reform. We consider a strong radiation filed $\left(\mathrm{G}_{0}=100\right.$, which corresponds to 100 times the ambient UV radiation field), and show that the efficiency of $\mathrm{O}_{2}$ formation is increased by a factor of 5 . On the other hand, the formation of $\mathrm{OH}$ and $\mathrm{H}_{2} \mathrm{O}$ is unchanged.

It is now possible to describe analytically the formation rates of chemical species on dust with simple formulae usable for any astrochemical model. These rates $\mathrm{k}_{\mathrm{OH} \text {,dust }}, \mathrm{k}_{\mathrm{H}_{2} \mathrm{O} \text {,dust }}$ and $\mathrm{k}_{\mathrm{O} 2 \text {,dust }}$ (in $\mathrm{cm}^{3} \mathrm{~s}^{-1}$ ) are reported in the supplementary material. The rate of the formation of species on dust (that are release in the gas) can be directly compared with gas phase rates in order to determine how molecules form in different environments with different physical conditions.

In the gas phase, the formation routes of water $\left(\mathrm{OH}+\mathrm{H}_{2}\right)$ and $\mathrm{OH}$ $\left(\mathrm{O}+\mathrm{H}_{2}\right)$ are associated with barriers of $1736 \mathrm{~K}, 3150 \mathrm{~K}$, respectively ${ }^{25}$. While the gas phase formation rate of $\mathrm{O}_{2}$ through $\mathrm{OH}+\mathrm{O}$, is still subject to debates (no barrier until $\mathrm{T}_{\text {gas }} \sim 150 \mathrm{~K}$ see references $\left.\mathrm{in}^{26}\right)$. The rates $\mathrm{k}_{\mathrm{OH} \text {,gas }}, \mathrm{k}_{\mathrm{H}_{2} \mathrm{O} \text {,gas }}\left(\right.$ in $\mathrm{cm}^{3} \mathrm{~s}^{-1}$ ) are reported in the supplementary material.

If we compare the formation rates of species via gas or dust routes, we need to compare $\mathrm{R}_{\text {gas }}=n_{X} \cdot n_{Y} \cdot k_{\text {gas }}$ to $\mathrm{R}_{\text {dust }}=n_{X} \cdot n_{H} \cdot k_{\text {dust }}$. In our case, $X$ is oxygen, since we are dealing with the formation of oxygenated species.

The formation of $\mathrm{OH}$ (and water) on dust surfaces dominates by 12 (9) orders of magnitude the gas phase route for a gas at $100 \mathrm{~K}$, while this becomes of $0(2)$ for a gas at $500 \mathrm{~K}$. These estimations are made considering the dust temperature in the range $(0-15 \mathrm{~K})$ for $\mathrm{OH}$ and $(0-25 \mathrm{~K})$ for water (and with the density of gas phase $\mathrm{O}$ and $\mathrm{OH}$ as $\mathrm{nOI}=10^{-4} \mathrm{n}_{H}, \mathrm{nOH}=10^{-7} \mathrm{n}_{H}$ ).

Searches of $\mathrm{O}_{2}$ with ground based telescopes and space missions such as SWASS and ODIN indicated that $\mathrm{O}_{2}$ has a widespread low abundance. $\mathrm{O}_{2}$ remained unseen allowing to derive some upper limits $\left(\mathrm{X}\left(\mathrm{O}_{2}\right) \leq 10^{-7}{ }^{27,28}\right)$. However, while recent Hershel observations confirmed the general trend that $\mathrm{O}_{2}$ has very low abundances (detection of $\mathrm{O}_{2}$ towards $\left.\rho \mathrm{Oph}, \mathrm{X}\left(\mathrm{O}_{2}\right) \sim 10^{-8}{ }^{29}\right)$, one isolated high abundance of $\mathrm{O}_{2}$ has been reported towards ORION ${ }^{26}$. This high abundance has been attributed to the presence of warm dust, or to shocks. In this work, our model shows an alternative efficient way to form $\mathrm{O}_{2}$ in the gas phase through chemical desorption from dust. This efficiency is important only for very specific conditions ( $\mathrm{T}_{\text {dust }} \sim 30-40 \mathrm{~K}$ and high UV field), which could explain why the detection of $\mathrm{O}_{2}$ is seen only in isolated cases. This will be investigated in a forthcoming paper.

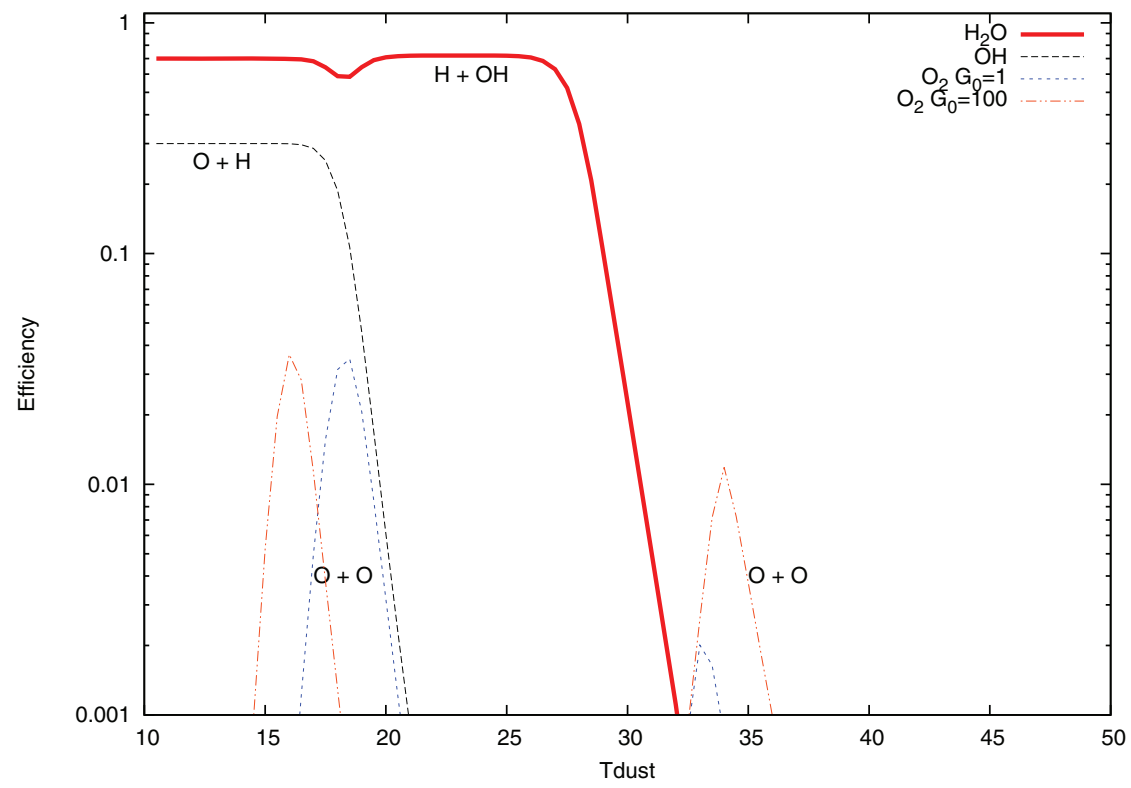

Figure $4 \mid$ Efficiency of chemical desorption as a function of dust temperature. This efficiency represents the fraction of oxygen released into the gas as $\mathrm{OH}, \mathrm{H}_{2} \mathrm{O}$ and $\mathrm{O}_{2}$. The first three reactions are computed for a UV field of $\mathrm{G}_{0}=1$, while the final three are for $\mathrm{G}_{0}=100$. Note that the amount of $\mathrm{O}_{2}$ released into the gas is significantly enhanced in the presence of a strong UV field. 
In summary, we report the first experimental evidence that species which form on the surface of interstellar dust grains can subsequently be released into the gas phase due to the exothermicity of the reaction. By depositing $\mathrm{O}_{2}$ molecules on several types of surfaces, and depositing $\mathrm{D}$ atoms in a second step, we show that an important fraction of the newly formed species are released into the gas phase during their formation, therefore confirming the existence and importance of the chemical desorption process. We also show that chemical desorption is sensitive to the type of surface upon which the formation reaction occurs. We derive canonical expressions for the formation of water, $\mathrm{OH}$ and $\mathrm{O}_{2}$ in the gas phase due to chemical desorption. These expressions can be implemented in any astrophysical model to properly account for the chemistry occurring in space. We show that this process, that covers gas phase species to another though dust, dominates by many orders of magnitude gas phase chemistry in the typical astrophysical environments where water and water ice are forming.

The process highlighted in this study, chemical desorption, has an important impact on the chemistry and thermodynamics of our Universe. In regions where stars are forming, the presence of dust dramatically influences the gas phase composition, changing the abundances of molecules available to cool the gas. Therefore, a cloud that undergoes gravitational collapse to form a star will cool more or less rapidly, depending on the available coolants, and this impacts the efficiency and characteristics (mass, binarity) of star formation. In this sense, the existence of chemical desorption, illustrated in this study, not only impacts the chemical composition of our Universe, but affects the way stars form.

\section{Methods}

Experimental setup. Experiments were performed using the FORMOLISM set-up ${ }^{13}$. In an ultra high vacuum chamber, a gold mirror covered with amorphous silicate (or a graphite substrate) is held at $10 \mathrm{~K}$. All the experiments are performed in the sub-monolayer regime (1 monolayer means that the surface is covered at $100 \%$ ), to ensure that the interactions of the species with the surface is not perturbed by layering effects. The surface coverage is determined using the specific desorption properties of the second layer ${ }^{15}$. One layer (or less) of $\mathrm{O}_{2}$ molecules is deposited at $10 \mathrm{~K}$. Coincidently, or just after $\mathrm{O}_{2}$ deposition, a $\mathrm{D}$ beam (with a dissociation efficiency of $70 \%$ ) is aimed at the solid sample covered with $\mathrm{O}_{2}$. In a second phase, the surface temperature is increased and the desorption flux is monitored using a movable quadrupole mass spectrometer, placed $3 \mathrm{~mm}$ away from the surface.

Microscopic model. To estimate the impact of chemical desorption on the gas phase composition of astrophysical environments, we use the chemical network presented in previous studies ${ }^{10,24}$, but include the chemical desorption yields derived from the experiments presented in this work (Table 1). We solve this chemical network using the rate equations method. We follow the evolution of the species on the surface in monolayers, and calculate the amount of newly formed species that desorb into the gas phase due to chemical desorption.

The main processes that are necessary to describe the chemistry occurring on a grain surface are described in details in this section. Here we enumerate these different processes: (1) species from the gas accrete on the dust (2) species on the dust diffuse on the surface (3) species on the surface react to form a product that is released in the gas phase (illustrated in fig. 1 with probabilities listed in table 1) (4) same than 3 but the product stays on he surface (5) evaporation of the surface species into the gas phase (6) photo-dissociation of surface species by UV photon. Our rate equation model follows the evolution of surface species with time. For this, we take into account the different processes occurring to the different species and calculate their associated rates. These processes are the following:

1) Species from the gas phase accrete onto the dust with a rate:

$$
R_{\text {acc }}=\frac{n_{X} v_{X} S}{n_{\text {site }}} \text { Monolayer s }^{-1},
$$

where $n_{X}$ and $v_{X}$ are, respectively, the gas phase density and velocity of the species $\mathrm{X}, \mathrm{n}_{\text {site }}$ is the density of sites on the dust $\left(10^{15} \mathrm{~cm}^{-2}\right)$, and $\mathrm{S}$ is the sticking coefficient of the species on the dust. We consider that $S=1$, meaning that when a species arrives on the dust, it directly sticks to the surface. 2) Species present on the surface evaporate from the surface with a rate:

$$
R_{\text {evap }(X)}=v_{X} \times \exp \left(-\frac{E_{X}}{k_{B} T}\right),
$$

where $v_{X}$ is the oscillation factor of the species (which is $10^{12} \mathrm{~s}^{-1}$ on physisorbed sites), and $\mathrm{E}_{X}$ is the binding energy of species $\mathrm{X}$, or in previous work ${ }^{30}$.
3) Species on the surface can diffuse on the surface with a mobility:

$$
\alpha_{X}=v_{X} \times\left(\exp \left(-0.4 a \sqrt{m_{X} E a_{X}}\right)+\exp \left(-\frac{E a_{X}}{k_{B} T}\right)\right) .
$$

The first exponential represents the diffusion occurring through tunneling, and the second through thermal hopping. $\mathrm{Ea}_{X}$ is the energy of the barrier between two physisorbed sites (which is considered here as $2 / 3$ of the binding energies $\mathrm{E}_{X}$ ), $a$ the width of the barrier (in $\AA$ ), and $\mathrm{m}_{X}$ the mass of the species $\mathrm{X}$ in amu. Note that the quantum tunneling is approximated ${ }^{20}$. Oxygen, even with its high mass, is observed to tunnel quite efficiently on the surface (Dulieu et al. submitted). We therefore adopt a barrier width of $1 \AA$ and barrier height of $500 \mathrm{~K}$ for the diffusion of oxygen on silicates. Oxygen can diffuse on the surface via quantum tunneling until $\mathrm{T}_{\text {dust }}=25 \mathrm{~K}$.

4) Species on the surface can be photodissociated by UV photons ${ }^{30}$ with a rate $\mathrm{R}_{\text {phot }} \mathrm{n}_{X}$.

5) Species on the surface can meet and react to form a product that will either stay on the surface or be released into the gas phase ${ }^{10}$.

The rate equation method couples the equations describing the different populations of the surface species. These equations are written as:

$\frac{d\left(X_{i}\right)}{d t}=R_{\text {acc } X_{i}}-X_{i} X_{j} \alpha_{X_{i}}+\left(1-f_{\text {des }}\right) X_{j} X_{k} \alpha_{X_{j}}-R_{\text {evapx }_{i}} X_{i}-R_{\text {phot }_{i}} X_{i}+R_{\text {phot }_{j}} X_{j}$,

where $X_{i}$ are the species on the surface. The six terms of this equation represent (1) the accretion of species $X_{i}$ from the gas phase, (2) the formation of new species that involve species $X_{i}$, (3) the creation of species $X_{i}$ by the encounter of species $X_{i}$ and $X_{j}$ that are present on the surface, (4) the evaporation of species $X_{i}$, (5) the photodissociation of $X_{i}$ on the dust, and (6) the creation of $X_{i}$ by photo-dissociation of species $Y$.

The formation of $\mathrm{OH}, \mathrm{H}_{2} \mathrm{O}$ and $\mathrm{O}_{2}$ are insured by several reactions routes on the dust but only the reaction $\mathrm{O}+\mathrm{H}, \mathrm{OH}+\mathrm{H}$ and $\mathrm{O}+\mathrm{O}$ see their products $\left(\mathrm{OH}, \mathrm{H}_{2} \mathrm{O}\right.$ and $\mathrm{O}_{2}$ ) released in the gas phase.The different gas phase efficiencies due to the chemistry on dust can be written as followed:

$$
\begin{gathered}
\epsilon_{\mathrm{OH}}=\frac{f d e s_{\mathrm{OH}} n_{O} n_{H} \alpha_{H}}{k_{a} c c(O)} \\
\epsilon_{\mathrm{H}_{2} \mathrm{O}}=\frac{f d e s_{\mathrm{H}_{2} \mathrm{O}} n_{\mathrm{OH}} n_{H \alpha_{H}}}{k_{a} c c(O)} \\
\epsilon_{\mathrm{O}_{2}}=\frac{2 f d e s_{\mathrm{O}_{2}} n_{O}^{2} \alpha_{O}}{k_{a} c c(O)}
\end{gathered}
$$

with fdes the chemical desorption fraction for the considered reaction as reported in table 2 .

Formation rates on dust due to chemical desorption. The formation rates of species $\mathrm{X}$ can be written as: $\mathrm{k}_{X, \text { dust }}=\mathrm{n}_{O} \mathrm{v}_{O} \mathrm{n}_{\text {dust }} \sigma \epsilon_{X}$, where $\mathrm{n}_{\text {dust }}$ is the density of dust, $\sigma$ the dust cross section and $\epsilon_{X}$ the efficiency of the formation of $\mathrm{OH}, \mathrm{H}_{2} \mathrm{O}$ or $\mathrm{O}_{2}$ (eq. 5, 6 and 7). If we consider a MRN grain size distribution $\left(n_{\text {dust }} \sigma / n(\mathrm{H})=10^{-21} \mathrm{~cm}^{-2}\right)$, then the rates for the formation of $\mathrm{OH}, \mathrm{H}_{2} \mathrm{O}$ and $\mathrm{O}_{2}$ are written as:

$$
\begin{aligned}
& k_{\mathrm{OH}, \text { dust }}=3.9 \cdot 10^{-17} \sqrt{\frac{T_{\text {gas }}}{100}} \epsilon_{\mathrm{OH}}\left(T_{\text {dust }}\right) \mathrm{cm}^{3} \mathrm{~s}^{-1} \\
&=3.9 \cdot 10^{-17} \sqrt{\frac{T_{\text {gas }}}{100}} \frac{0.3}{1+2 \cdot 10^{21} \frac{n O I}{n H I^{2}} \exp -\frac{1100}{T_{\text {dust }}}} \mathrm{cm}^{3} \mathrm{~s}^{-1} \\
& k_{\mathrm{H}_{2} \mathrm{O}, \text { dust }}= 3.9 \cdot 10^{-17} \sqrt{\frac{T_{\text {gas }}}{100}} \epsilon_{\mathrm{H}_{2} \mathrm{O}}\left(T_{\text {dust }}\right) \mathrm{cm}^{3} \mathrm{~s}^{-1} \\
&= 3.9 \cdot 10^{-17} \sqrt{\frac{T_{\text {gas }}}{100}} \frac{0.7}{1+2 \cdot 10^{21} \frac{n O I}{n H I^{2}} \sqrt{\frac{100}{T_{\text {gas }}}} \exp -\frac{833}{T_{\text {dust }}}} \cdot \mathrm{cm}^{3} \mathrm{~s}^{-1} \\
& k_{\mathrm{O}_{2}, \text { dust }}=1.9 \cdot 10^{-17} \sqrt{\frac{T_{\text {gas }}}{100}} \epsilon_{\mathrm{O}_{2}}\left(T_{\text {dust }}\right) \mathrm{cm}^{3} \mathrm{~s}^{-1} \\
&=1.9 \cdot 10^{-17} \sqrt{\frac{T_{\text {gas }}}{100}} \frac{1.3 \cdot 10^{-23} n O I \sqrt{\frac{T_{\text {gas }}}{100}} \cdot \exp \frac{-500}{T_{\text {dust }}}}{d e n^{2}} \\
& \mathrm{~cm}^{3} \mathrm{~s}^{-1},
\end{aligned}
$$

where $\mathrm{nHI}$ and $\mathrm{nOI}$ are the densities of atomic hydrogen and oxygen in the gas phase, respectively. The rate for the formation efficiency of $\mathrm{O}_{2}$ is complex, and the denominator of the expression (den) is written as: 


$$
\text { den }=1.5 \cdot 10^{22} \cdot n_{H I} \cdot \sqrt{\frac{T_{\text {gas }}}{100}} \cdot \exp \frac{143}{T_{\text {dust }}}+\exp \frac{-1076}{T_{\text {dust }}}+\exp \frac{-500}{T_{\text {dust }}} \cdot \mathrm{OH}
$$

With $\mathrm{OH}$ the approximate of the $\mathrm{OH}$ surface species on the dust:

$$
\mathrm{OH}=\frac{0.26 \frac{n O I}{n H I} \cdot \exp \frac{-143}{T_{\text {dust }}}}{1+\frac{0.6 \cdot 10^{22} \exp \frac{-1343}{T_{\text {dust }}}}{n H I \sqrt{\frac{T_{\text {gas }}}{100}}}}
$$

As the temperature increases, some reactants evaporate and we enter a stochastic regime where the system should be treated with stochastic methods (Monte Carlo). Therefore, the rates we derive here overestimate the actual rates at temperatures greater than $30 \mathrm{~K}^{24}$ (when the rates decrease exponentially). Note that the value of $\mathrm{T}_{\text {dust }}$ for the validity of our estimates is higher at higher densities.

Formation rates in the gas phase. In the gas phase, the formation routes of water $\left(\mathrm{OH}+\mathrm{H}_{2}\right)$ and $\mathrm{OH}\left(\mathrm{O}+\mathrm{H}_{2}\right)$ are associated with barriers of $1736 \mathrm{~K}, 3150 \mathrm{~K}$ respectively ${ }^{25}$. These rates can be written as:

$$
\begin{aligned}
& k_{\mathrm{OH}, \text { gas }}=3.14 \cdot 10^{-13}\left(\frac{T_{\text {gas }}}{300}\right)^{2.70} \exp \left(-\frac{3150}{T_{\text {gas }}}\right) \mathrm{cm}^{3} \mathrm{~s}^{-1} \\
& k_{\mathrm{H}_{2} \mathrm{O}, \text { gas }}=2.05 \cdot 10^{-12}\left(\frac{T_{\text {gas }}}{300}\right)^{1.52} \exp \left(-\frac{1736}{T_{\text {gas }}}\right) \mathrm{cm}^{3} \mathrm{~s}^{-1} .
\end{aligned}
$$

1. Trumpler, R. J. Absorption of Light in the Galactic System. PASP 42, 214 (1930).

2. Oort, J. H. \& van de Hulst, H. C. Gas and smoke in interstellar space. Bull. Astron. Inst. Netherlands 10, 187 (1946).

3. Walmsley, C., Interstellar Dust, Allamandola, L. J. \& Tielens, A. G. G. M. eds. (1989), vol. 135 of IAU Symposium, 263.

4. Kristensen, L. E. et al. Methanol maps of low-mass protostellar systems. I. The Serpens molecular core. A\& A 516, A57 (2010).

5. Takahashi, J. \& Williams, D. A. Chemically driven desorption of CO from icy grains in dark clouds. MNRAS 314, 273 (2000)

6. Andersson, S., Al-Halabi, A., Kroes, G.-J. \& van Dishoeck, E. F. Moleculardynamics study of photodissociation of water in crystalline and amorphous ices. Journal of Chemical Physics 124, 064715 (2006).

7. Morisset, S., Aguillon, F., Sizun, M. \& Sidis, V. Quantum dynamics of $\mathrm{H}_{2}$ formation on a graphite surface through the Langmuir Hinshelwood mechanism. Journal of Chemical Physics 121, 6493 (2004).

8. Bergeron, H., Rougeau, N., Sidis, V., Sizun, M., Teillet-Billy, D. \& Aguillon, F. OH Formation from O and H Atoms Physisorbed on a Graphitic Surface through the Langmuir Hinshelwood Mechanism: A Quasi Classical Approach. Journal Phys. Chem. A 112, 11921 (2008).

9. Garrod, R. T., Wakelam, V. \& Herbst, E. Non-thermal desorption from interstellar dust grains via exothermic surface reactions. A\&A 467, 1103 (2007).

10. Cazaux, S., Cobut, V., Marseille, M., Spaans, M. \& Caselli, P. Water formation on bare grains: When the chemistry on dust impacts interstellar gas. A\&A 522, A74+ (2010).

11. Pirronello, V., Liu, C., Shen, L. \& Vidali, G. Laboratory Synthesis of Molecular Hydrogen on Surfaces of Astrophysical Interest . ApJL 475, L69+ (1997).

12. Pirronello, V., Liu, C., Roser, J. E. \& Vidali, G. Measurements of molecular hydrogen formation on carbonaceous grains. AઐA 344, 681 (1999).

13. Amiaud, L., Dulieu, F., Baouche, S., Fillion, J. H., Momeni, A. \& Lemaire, J. L. Isotopic Segregation of Molecular Hydrogen on Water Ice Surface at Low Temperature: Importance for Interstellar Grain Chemistry. eds. (2006), vol. 855 of American Institute of Physics Conference Series, 55-61.

14. Congiu, E., Matar, E., Kristensen, L. E., Dulieu, F. \& Lemaire, J. L. Laboratory evidence for the non-detection of excited nascent $\mathrm{H}_{2}$ in dark clouds. MNRAS 397, L96 (2009).
15. Noble, J. A. et al. The desorption of $\mathrm{H}_{2} \mathrm{CO}$ from interstellar grains analogues. A\&A 543, A5 (2012).

16. Miyauchi, N., Hidaka, H., Chigai, T., Nagaoka, A., Watanabe, N. \& Kouchi, A. Formation of hydrogen peroxide and water from the reaction of cold hydrogen atoms with solid oxygen at $10 \mathrm{~K}$. Chemical Physics Letters 456, 27 (2008).

17. Ioppolo, S., Cuppen, H. M., Romanzin, C., van Dishoeck, E. F. \& Linnartz, H. Laboratory Evidence for Efficient Water Formation in Interstellar Ices. ApJ 686, 1474 (2008).

18. Dulieu, F. et al. Experimental evidence for water formation on interstellar dust grains by hydrogen and oxygen atoms. A\&A 512, A30 (2010).

19. Pettersson, M., Tuominen, S. \& Räsänen, M. IR Spectroscopic Study of $\mathrm{H}_{2} \mathrm{O}_{2}$, $\mathrm{HDO}_{2}$, and $\mathrm{D}_{2} \mathrm{O}_{2}$ Isolated in $\mathrm{Ar}, \mathrm{Kr}$, and Xe Matrices. JPC A 101 (6), 1166-1171 (1997).

20. Cazaux, S. \& Tielens, A. G. G. M. $\mathrm{H}_{2}$ Formation on Grain Surfaces. ApJ 604, 222 (2004).

21. Fraser, H. J., Collings, M. P., McCoustra, M. R. S. \& Williams, D. A. Thermal desorption of water ice in the interstellar medium. MNRAS 327, 1165 (2001)

22. Jing, D., He, J., Brucato, J., De Sio, A., Tozzetti, L. \& Vidali, G. On Water Formation in the Interstellar Medium: Laboratory Study of the O + D Reaction on Surfaces. ApJL 741, L9 (2011).

23. Congiu, E., Chaabouni, H., Laffon, C., Parent, P., Baouche, S. \& Dulieu, F. Efficient surface formation route of interstellar hydroxylamine through $\mathrm{NO}$ hydrogenation. I. The submonolayer regime on interstellar relevant substrates. JCP 137, 054713 (2012)

24. Meijerink, R., Cazaux, S. \& Spaans, M. Enhanced $\mathrm{H}_{2} \mathrm{O}$ formation through dust grain chemistry in X-ray exposed environments. A\&A 537, A102 (2012).

25. Manion, J. A. et al. NIST Chemical Kinetics Database NIST Standard Reference Database 17, Version 7.0 (Web Version), Release 1.4.3. Data version 2008.12, National Institute of Standards and Technology, Gaithersburg, Maryland, 20899 8320. Web address: http://kinetics.nist.gov/ (2008).

26. Goldsmith, P. F. et al. Herschel Measurements of Molecular Oxygen in Orion. ApJ 737, 96 (2011).

27. Goldsmith, P. F. et al. $\mathrm{O}_{2}$ in Interstellar Molecular Clouds. ApJL 539, L123 (2000)

28. Pagani, L. et al. Low upper limits on the $\mathrm{O}_{2}$ abundance from the Odin satellite. AঊA 402, L77 (2003).

29. Liseau, R. et al. Multi-line detection of $\mathrm{O}_{2}$ toward $\rho$ Ophuichi A. AઐA 541, A73 (2012)

30. Cuppen, H. M. \& Herbst, E. Simulation of the Formation and Morphology of Ice Mantles on Interstellar Grains. ApJ 668, 294 (2007).

\section{Acknowledgements}

S.C. is supported by the Netherlands Organization for Scientific Research (NWO), J.A.N thanks The Leverhulme Trust and the Scottish International Education Trust and Royal Commission for the Exhibition of 1851 Research Fellow for fundings.

\section{Author contributions}

S. Cazaux and F. Dulieu wrote the main manuscript. The experiements were perfomed by E. Congiu, J. A. Noble, S. Baouche, H. Chaabouni, A. Moudens, M. Minissale and F. Dulieu. S. Cazaux developed the theoretical model. All authors reviewed the manuscript.

\section{Additional information}

Competing financial interests: The authors declare no competing financial interests.

License: This work is licensed under a Creative Commons

Attribution-NonCommercial-NoDerivs 3.0 Unported License. To view a copy of this license, visit http://creativecommons.org/licenses/by-nc-nd/3.0/

How to cite this article: Dulieu, F. et al. How micron-sized dust particles determine the chemistry of our Universe. Sci. Rep. 3, 1338; DOI:10.1038/srep01338 (2013). 